\title{
Mythos
}

Rivista di Storia delle Religioni

$14 \mid 2020$

Varia

\section{La fin mystérieuse d'CEdipe dans l'ÆEdipe à Colone de Sophocle : aspects religieux et enjeux dramatiques d'une héroïsation}

The Mysterious Death of Edipus in Sophocles' Edipus at Colonus: Religious Aspects and Dramatic Meaning of CEdipus' Heroization.

\section{Christine Mauduit}

\section{OpenEdition}

Journals

Édition électronique

URL : https://journals.openedition.org/mythos/2255

DOI : $10.4000 /$ mythos.2255

ISSN : 2037-7746

Éditeur

Salvatore Sciascia Editore

\section{Référence électronique}

Christine Mauduit, «La fin mystérieuse d'EEdipe dans I'Éfipe à Colone de Sophocle : aspects religieux et enjeux dramatiques d'une héroïsation », Mythos [En ligne], 14 | 2020, mis en ligne le 31 décembre 2020, consulté le 06 octobre 2021. URL : http://journals.openedition.org/mythos/2255 ; DOI : https:// doi.org/10.4000/mythos.2255

Ce document a été généré automatiquement le 6 octobre 2021.

Mythos 


\title{
La fin mystérieuse d'œdipe dans l'CEdipe à Colone de Sophocle : aspects religieux et enjeux dramatiques d'une héroïsation
}

\author{
The Mysterious Death of EEdipus in Sophocles' Edipus at Colonus: Religious \\ Aspects and Dramatic Meaning of CEdipus' Heroization.
}

Christine Mauduit

1 L'emploi moderne du mot " héros », dans son application à un personnage de tragédie, est marqué au sceau de l'ambiguïté, du fait qu'il peut recouvrir trois sens, qui sont à la fois distincts et indissociables. Le mot sert d'abord, au sens littéraire, à préciser le statut du personnage dans l'œuvre et il désigne le protagoniste de la pièce, celui dont les malheurs et les revirements de fortune constituent le sujet de la fiction tragique (héros au sens 1). Il s'applique ensuite, en un sens que l'on qualifiera de mythologique, à un personnage légendaire marqué par un destin exceptionnel, qui se manifeste en général dès sa naissance, s'accomplit dans une existence hors-normes et s'achève par une fin qui consacre, d'une manière ou d'une autre, sa différence par rapport à l'humanité ordinaire (héros au sens 2). Il est employé enfin, en un sens religieux, pour désigner un personnage à qui l'on a institué un culte après sa mort, généralement en récompense de services rendus pendant son existence (héros au sens 3). On voit bien le lien qui existe entre ces différentes acceptions dans le contexte d'une tragédie. C'est parce qu'il est un héros au sens 2 qu'un personnage est choisi comme protagoniste d'une tragédie (sens 1); et c'est aussi parce qu'il est un héros au sens 2 qu'il peut devenir un héros au sens 3 et recevoir un culte, qui, dans certains cas au moins - et c'est là la coloration proprement tragique du phénomène - est perçu comme une forme de compensation pour une vie de souffrances.

2 Les deux premiers sens précédemment définis pour le mot héros sont clairement pertinents à propos d'Ædipe dans $\Subset$ dipe à Colone : il est à la fois le protagoniste de la tragédie et un personnage mythologique marqué par un destin exceptionnel. C'est 
cependant le troisième sens ou, plus précisément, l'articulation entre le deuxième et le troisième sens qui est principalement en question lorsque l'on parle de l'héroïsation d'œedipe. Si la critique a noté, depuis longtemps, qu'il s'agit là du thème central de la pièce ${ }^{1}$, la question qui reste discutée est de savoir si, et jusqu'à quel point la mort mystérieuse d'œedipe dans le bois sacré des Euménides doit être mise en rapport avec les données et les catégories de pensée de la religion grecque, en l'occurrence, les cultes rendus à đEdipe en Attique et, de façon plus générale, avec les composantes et le modèle du culte héroïque. C'est à ce débat que l'exposé suivant voudrait contribuer.

Rappelons brièvement l'argument de la pièce. Représentée en 401 av. J.-C., quelques années après la mort du poète, par les soins de son petit-fils Sophocle le Jeune, cette tragédie met en scène une version originale de la fin d'ÆEdipe, selon laquelle le héros, au terme d'une vie d'errance et de mendicité, consécutive à la découverte de ses crimes, parvient à Colone, aux portes d'Athènes, sous la conduite de sa fille Antigone. Ayant pénétré sans le savoir dans le bois sacré des Euménides, il découvre qu'il s'agit du lieu qu'un oracle d'Apollon lui a désigné comme devant être celui de son dernier séjour. D'abord rejeté par les Coloniates, il est accueilli par Thésée, le roi d'Athènes, qui le prend sous sa protection et empêche Créon de le ramener de force à Thèbes. Après une ultime entrevue avec son fils Polynice, venu le supplier de prendre son parti dans la guerre qui l'oppose à son frère, ๔edipe meurt d'une mort mystérieuse, qui le transforme en un héros puissant, protecteur de l'Attique et ennemi des Thébains. La pièce combine ainsi une dimension politique - l'intégration à Athènes d'un héros thébain - et une dimension religieuse - le passage d'€́dipe à un statut supra-humain - dans la représentation des derniers moments de la vie d'ÆEdipe. Bien que ces deux aspects soient étroitement liés l'un à l'autre, c'est le second qui nous retiendra surtout ici, compte tenu du thème proposé à notre réflexion dans le cadre de ce colloque.

Edipe à Colone n'est assurément pas la seule tragédie qui s'achève sur la perspective de l'héroïsation du protagoniste et de l'instauration d'un culte en son honneur'2. Dans le corpus de Sophocle, la partie finale d'Ajax - avec, en particulier, la référence à la protection que le cadavre du héros offre aux siens ${ }^{3}$ - a été lue par certains critiques à la lumière du culte héroïque ${ }^{4}$ et une approche du même type a également été proposée

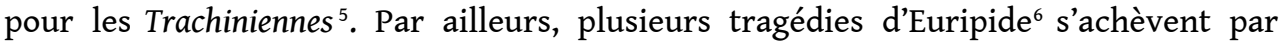
l'intervention d'un deus ex machina (voire d'un personnage humain) qui annonce l'instauration, dans un futur extra-dramatique, d'un culte expressément mis en relation avec les événements représentés dans la tragédie. Mais ces étiologies finales ont surtout pour fin d'établir un lien entre le passé mythique et le présent des spectateurs. Le thème de l'héroïsation ne joue en revanche aucun rôle significatif pour l'interprétation d'ensemble de ces pièces.

5 Il en va tout autrement dans le cas d' $\Subset$ dipe à Colone, car c'est ici la tragédie tout entière qui préfigure et dramatise l'accession finale d'ÆEdipe au statut de héros. Même si l'existence de cultes rendus à đEdipe en Attique a pu jouer un rôle dans la conception de cette fin d'ÆEdipe, la pièce de Sophocle ne peut donc pas être lue simplement comme une sorte de récit étiologique destiné à expliquer les cultes rendus à đEdipe en Attique ; une difficulté supplémentaire pour qui veut emprunter cette voie tient au fait que la chronologie de ces cultes et, partant, leur existence même à l'époque de Sophocle sont mal établies, ainsi que nous le verrons dans la première partie de cet exposé. C'est le point d'intersection entre la représentation tragique et la réalité cultuelle de l'hérö̈sation que je chercherai à cerner dans un second temps, sans prétendre épuiser 
par là la signification de cette transformation d'ÆEdipe, qui ne peut être pleinement saisie que sur la base d'une interprétation dramatique de la pièce ${ }^{7}$. Il est nécessaire, pour pouvoir procéder à cette lecture et apprécier pleinement la portée et la signification des choix de Sophocle, de présenter d'abord, de façon synthétique, les différentes traditions relatives à la mort et aux cultes d'œEdipe. Peut-être peut-on espérer parvenir, à l'issue de ces quelques réflexions, à une position plus nuancée sur le rôle de cet arrière-plan cultuel que celle qui consiste à y voir la clé de lecture de la pièce $^{8}$ ou celle qui, a contrario, lui dénie toute pertinence pour son interprétation 9 .

\section{Le « dossier » Édipe ${ }^{10}$ et le muthos d'E्Edipe à Colone}

\section{Les traditions mythiques relatives à la mort d'CEdipe}

6 En choisissant de faire mourir đEdipe à Colone, Sophocle s'écarte de la tradition la plus ancienne, qui situe à Thèbes le lieu de la mort et des funérailles d'ÆEdipe. Cette version thébaine est attestée pour la première fois dans un passage du chant XXIII de l'Iliade, qui évoque les jeux organisés à l'occasion des funérailles de Patrocle. Il y est question d'Euryale, l'un des participants à l'épreuve de pugilat qui « vint jadis à Thèbes pour les funérailles d'ÆEdipe, abattu » et «remporta la victoire sur tous les descendants de Cadmos » ${ }^{11}$. L'emploi du participe $\delta \varepsilon \delta o u \pi$ ótoc, littéralement " tombé avec fracas ${ }^{12}$ " indique qu'CEdipe est mort dans un combat (bien qu'aucune tradition mythique ne puisse éclairer cette allusion) et l'évocation des jeux donnés à l'occasion de ses funérailles indique qu'il a été enterré à Thèbes avec tous les honneurs. Ce sont les funérailles ordinaires des héros de l'Iliade et l'insertion de l'allusion dans le récit des jeux funèbres en l'honneur de Patrocle est significative à cet égard.

7 Cette version épique de la mort d'œEdipe survit dans plusieurs tragédies du cycle thébain. Elle est à l'arrière-plan de deux passages des Sept contre Thèbes, où le chœur signale qu'Étéocle et Polynice, qui viennent de s'entretuer, vont partager, à Thèbes, la tombe de leur père ${ }^{13}$. Le premier de ces deux passages mentionne, en passant, la tombe d'ÆEdipe à Thèbes, mais sans donner de détail sur sa mort, puisque c'est le meurtre mutuel de ses fils qui est au centre de la tragédie; il s'agit surtout de souligner la communauté de destin entre le père et les fils. La version thébaine est également celle que suit Sophocle dans Antigone, lorsqu'il fait dire à l'héroïne qu'elle a accordé les honneurs funèbres à son père, à sa mère et à son frère (Étéocle) ${ }^{14}$.

C'est dans les versions non thébaines, qui sont attestées postérieurement, que l'on voit apparaître des idées nouvelles sur la mort et la sépulture d'œdipe. Elles sont liées à la représentation du héros comme un criminel souillé, dont le contact doit, pour cette raison, être évité de son vivant, mais aussi après sa mort. Les différents récits qui relèvent de cette autre branche de la tradition font intervenir, d'une manière ou d'une autre, la thématique de l'exil et de l'errance d'EEdipe, et font état de problèmes ou de conflits suscités par son ensevelissement. L'une de ces versions, due à Lysimaque d'Alexandrie (ca 200 av. J.-C.) et transmise dans une scholie au v. 91 d' Edipe à Colone comme une tradition alternative à la version coloniate, raconte toutes les vicissitudes survenues au cadavre d'Ædipe avant qu'il soit finalement enseveli dans un sanctuaire de Déméter à Étéonos, en Béotie ${ }^{15}$. Je la résume dans ses grandes lignes : après la mort d'Æ̇dipe, les Thébains refusent qu'il soit enterré à Thèbes, parce qu'ils le considèrent comme souillé ( $\dot{\alpha} \sigma \varepsilon \beta o \tilde{\varsigma})$; ses amis l'enterrent dans une bourgade de Béotie nommée 
Céos, mais comme des malheurs surviennent, les habitants de Céos les attribuent à la présence de la sépulture d'ÆEdipe et leur ordonnent de déplacer le cadavre. Les amis

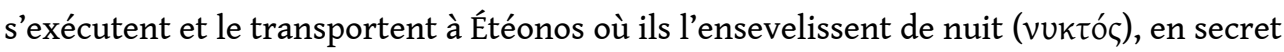
( $\left(\alpha^{\prime} \theta \rho \alpha \underline{)}\right)$, mais sans s'en rendre compte, dans un sanctuaire de Déméter. Lorsqu'ils le découvrent, ils vont consulter Apollon, qui leur donne l'ordre de ne pas déplacer la

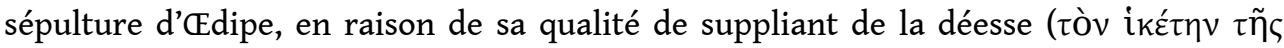
$\theta \varepsilon \circ \tilde{\text { ) }}$. Le sanctuaire (ou sans doute la sépulture d'ÆEdipe dans le sanctuaire) reçoit le nom d'œEdipodeion.

De ce récit, on retiendra principalement les éléments suivants :

- le fait que la souillure qui s'attachait à đedipe de son vivant reste attachée à son cadavre, qui est considéré comme une source de malheurs pour les gens du pays où se trouve sa sépulture.

- la nécessité où se trouvent ses amis de l'enterrer en secret.

- le fait que le cadavre d'ÆEdipe a été déplacé à deux reprises : de Thèbes, où đEdipe est mort, à Céos, puis de Céos, où il a été enseveli une première fois, à Étéonos, où il se fixe enfin et où un tombeau lui est érigé. Il y a donc, si l'on peut dire, exil et errance du cadavre d'CEdipe.

- le fait que son tombeau ( $\mu \nu \tilde{\eta} \mu \alpha)$ se trouve à l'intérieur d'un sanctuaire de Déméter, à Étéonos.

Tous ces éléments, ainsi que le nom d'ÆEdipodeion donné au lieu de la sépulture d'Æ̇dipe, inclinent à penser que l'on a ici un récit étiologique lié à un culte local d'CEdipe.

La version qui situe à Colone, en Attique, la mort d'Ædipe est rapportée, en dehors de Sophocle ${ }^{16}$, par l'Atthidographe Androtion ${ }^{17}$, sans que l'on soit en mesure d'établir un lien de dépendance entre ces deux témoignages (Androtion dépend-il de Sophocle ? Les deux auteurs dépendent-ils d'une source commune perdue ? $\left.^{18}\right)$. On peut à tout le moins souligner la grande proximité entre les deux récits. D'après le témoignage d'Androtion, ๔dipe, chassé de Thèbes par Créon, arrive en Attique, au lieu dit Colonos Hippeus. Il se réfugie en suppliant dans le sanctuaire des déesses Déméter et Athéna Poliouchos. Créon cherche à l'emmener de force, mais Thésée prend sa défense. Au moment où il va mourir, ๔dipe demande à Thésée de ne révéler à personne le lieu de sa tombe ( $\mu \eta \delta \varepsilon v i$

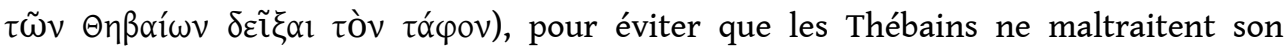

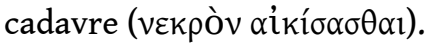

11 Cette version évoque, selon un schéma très proche de celui de la tragédie sophocléenne, les tentatives déployées par Créon pour s'emparer d'ÆEdipe et l'intervention salvatrice de Thésée. Elle fait aussi état d'un secret entretenu, à la demande d'œEdipe, autour de l'emplacement de sa sépulture. Ces éléments supposent l'attribution à la personne et au cadavre d'ÆEdipe d'un certain pouvoir qu'il s'agit de neutraliser. Les mauvais traitements qu'Ædipe redoute se réfèrent sans doute aux mutilations que ses ennemis pourraient infliger à son cadavre pour l'empêcher de leur nuire et de se venger (on songe aux mutilations infligées par Clytemnestre au cadavre d'Agamemnon). Les principales différences avec la version mise en scène par Sophocle résident d'une part dans le fait que les divinités auprès desquelles đEdipe se réfugie en suppliant sont Déméter et Athéna Poliouchos, et non les Euménides ; d'autre part, dans le fait qu'€dipe meurt de vieillesse, c'est-à-dire de la mort la plus ordinaire qui soit ; enfin, dans le fait que s'il semble pouvoir nuire à ses ennemis après sa mort, il ne 
semble exercer, en revanche aucun pouvoir salvateur au bénéfice de Thésée et de l'Attique.

\section{Les cultes d'EEdipe en Attique}

12 En dehors des traditions mythiques relatives à la mort d'œEdipe, l'enquête sur l'arrièreplan religieux et les sources d'inspiration de la tragédie de Sophocle doit prendre en compte les données relatives aux cultes rendus à đedipe en Attique. Notre principale source sur ce point est Pausanias. Quand il visite la région, ce n'est pas à Colone, mais à Athènes même, à l'intérieur du sanctuaire des Vénérables (Semnai) - c'est-à-dire des Euménides - sur l'Aréopage, qu'il voit le tombeau d'ÆEdipe :

Auprès, il y a le sanctuaire des déesses que les Athéniens appellent Semnai (Vénérables) mais qu'Hésiode, dans sa Théogonie, appelle Érinyes [...]. À l'intérieur

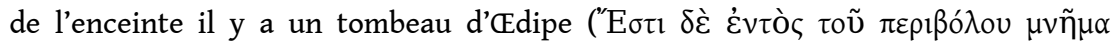

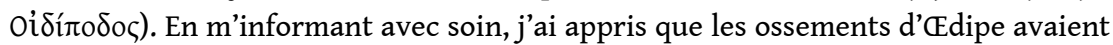

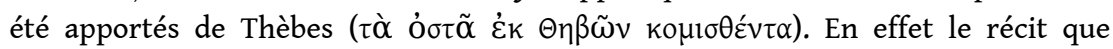

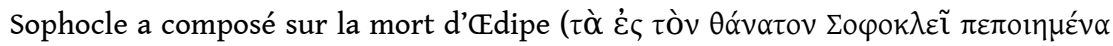

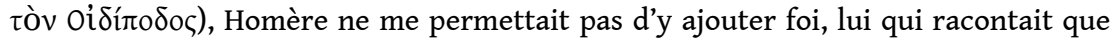
Mécistée est venu à Thèbes pour prendre part au concours des jeux funèbres à la mort d'ÆEdipe. ${ }^{19}$

Pausanias, qui connaît ses classiques, oppose la version de Sophocle et celle d'Homère sur le lieu de la mort d'ÆEdipe. Accordant plus de foi à l'auteur de l'Iliade, il cherche à concilier la version homérique avec les faits religieux dont portent témoignage les vestiges archéologiques ${ }^{20}$ et " découvre » en enquêtant une tradition selon laquelle les ossements d'ÆEdipe auraient été transférés de Thèbes à Athènes ${ }^{21}$. On notera qu'il ne fait pas expressément état des autres versions non thébaines, mais l'enquête qu'il dit avoir

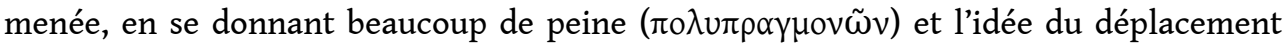
des ossements d'œEdipe de Thèbes à Colone pourraient laisser penser qu'il a eu accès au récit de Lysimaque, et qu'il l'a adapté à la version attique. Ce qu'il convient de retenir surtout, c'est l'association d'œdipe et des Euménides : le tombeau d'œdipe se trouve à l'intérieur du sanctuaire des déesses.

Un peu plus loin, le Périégète donne d'autres informations, relatives cette fois aux cultes de Colone, et il fait mention, dans ce contexte, d'un sanctuaire héroïque consacré conjointement à Pirithoos, Thésée, CEdipe et Adraste.

On montre aussi un endroit appelé Colonos Hippios, lieu de l'Attique où l'on dit

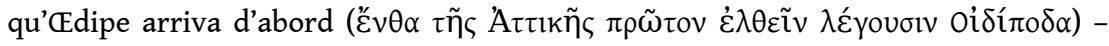
tradition différente de celle que l'on trouve chez Homère, mais qui a cours cependant ; [on y montre aussi] un autel de Poséidon Hippios et d'Athéna Hippia, un sanctuaire héroïque de Pirithoüs et de Thésée, d'œdipe et d'Adraste - $\dot{\eta} \rho \tilde{\omega}$ ov $\delta \grave{\varepsilon}$

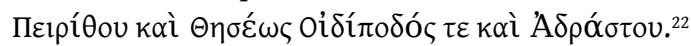

Certaines des réalités évoquées par le Périégète dans ce passage concordent avec le paysage religieux de Colone dépeint par Sophocle au début de sa tragédie. Le premier Coloniate qui découvre la présence d'Antigone et d'ÆEdipe leur révèle le caractère sacré du lieu et cite Poséidon parmi les divinités auxquelles il est consacré (v. 54-55) ; il leur explique ensuite que Colone doit son nom au héros cavalier Colonos (Colonos Hippios), dont une statue est sans doute visible dans l'espace scénique (v. 58-61). L'association d'ÆEdipe avec Thésée et Pirithoos représente un autre point de contact entre $E$ dipe à Colone et Pausanias. Dans le récit qu'il fait de la disparition miraculeuse d'€Edipe, le messager signale, en effet, qu'en se dirigeant vers le lieu de sa mort, ÆEdipe est passé 
près d'un cratère contenant le texte des serments que s'étaient jadis échangés Thésée et Pirithoos (sc. avant de descendre aux Enfers pour enlever Perséphone) ${ }^{23}$. Le poète inclut donc, dans la topographie sacrée de la mort d'œedipe, un lieu mémoriel dédié à Thésée et Pirithoos.

On notera cependant que Pausanias, qui vient de signaler la présence d'un tombeau d'ÆEdipe dans le sanctuaire des Euménides à Athènes, ne fait pas expressément état d'un tombeau d'ÆEdipe à Colone. Son témoignage n'associe pas non plus ÆEdipe aux déesses Euménides, mais à d'autres héros, avec lesquels sa légende est plus ou moins directement liée.

Le témoignage de Pausanias nous met donc en présence de deux lieux de culte d'ÆEdipe en Attique, faiblement distants l'un de l'autre: un tombeau - $\mu v \tilde{\eta} \mu \alpha-$ dans le sanctuaire des Euménides à Athènes, et un hérôon collectif, où đEdipe semble avoir été plus précisément associé à Adraste ${ }^{24}$.

Mais qu'en était-il de ces réalités cultuelles à l'époque de Sophocle?

Les savants ont beaucoup discuté pour savoir lequel de ces deux cultes - celui d'Athènes ou celui de Colone - était antérieur à l'autre, et se sont demandé s'il était possible qu'un culte ait été rendu à đEdipe à la même époque dans deux lieux aussi proches de l'Attique. Certains ${ }^{25}$ arguant du fait qu'il n'y a, avant ÆEdipe à Colone, aucun témoignage attestant l'existence d'un lien entre ÆEdipe et Colone, tendent à penser que Sophocle est l'inventeur de la version coloniate, et que l'idée d'associer Edipe et les Euménides à Colone lui a été inspirée par l'association d'ÆEdipe et des Euménides sur l'Aréopage. D'autres, plus nombreux (Kamerbeek ${ }^{26}$, Jouanna ${ }^{27}$, Kearns ${ }^{28}$ ) font valoir au contraire que Sophocle n'aurait pas situé la mort d'ÆEdipe à Colone si ses contemporains avaient su que la tombe d'ÆEdipe se trouvait à Athènes sur l'Aréopage et considèrent que c'est le culte de Colone qui est le plus ancien. D'autres encore (Jebb ${ }^{29}$ suivi par Kelly ${ }^{30}$ ) supposent que le secret qui entoure la tombe d'ÆEdipe, dans la pièce de Sophocle - point sur lequel nous reviendrons dans le deuxième temps de cet exposé -, rend compte des incertitudes qui existaient, à son époque, sur le lieu de la mort du héros. Au vu de l'ensemble des éléments du dossier, j'aurais tendance, pour ma part, à privilégier l'idée d'une coexistence à l'époque de Sophocle, sinon de cultes actifs, du moins de traditions mythiques divergentes sur la mort d'Ædipe et à supposer que Sophocle a choisi - de préférence à la version athénienne - une tradition locale coloniate (qui n'était peut-être pas la plus connue à son époque ${ }^{31}$ ), pour rendre hommage à sa patrie. Au demeurant, il convient, pour minimiser l'opposition entre la version athénienne et la version coloniate, de rappeler à quel point Colone et Athènes sont liées dans la tragédie de Sophocle ${ }^{32}$.

Telles sont donc, présentées dans leurs grandes lignes, les composantes de la tradition mythique et les données cultuelles relatives à đEdipe, sur fond desquelles Sophocle compose sa tragédie. Redisons que les questions de chronologie restent indécidables et qu'il n'est pas possible de savoir précisément quels étaient l'état des mythes et la réalité du culte d'CEdipe au moment où le poète a composé sa pièce, ni, a contrario, quelle influence la représentation de la fin d'Edipe dans Edipe à Colone a pu avoir sur l'évolution ultérieure de ces données. Les points de contact que nous avons relevés, chemin faisant, entre le muthos mis en scène par Sophocle et tel ou tel témoignage suggèrent que le poète a puisé, de façon éclectique, dans cette "nébuleuse œedipienne » (cf. en particulier l'association d'Ædipe et des Euménides ou encore l'idée d'un secret entretenu autour du lieu de sa mort), qu'il s'est inspiré de traditions antérieures, mais 
qu'il les a réélaborées et recomposées dans un drame original, dont le sens ne réside pas dans le rapport qu'il entretient avec ces données extérieures. Ainsi, même s'il existait, à son époque, un culte rendu à Ædipe, à Athènes et/ou à Colone, il ne paraît pas possible de ramener la tragédie de Sophocle à une sorte d'étiologie de ce culte et ce, d'autant moins qu'il n'y est pas fait mention de rites qui doivent être régulièrement accomplis sur la tombe du héros ${ }^{33}$. Le poète donne de même une signification symbolique à l'association d'€dipe et des Euménides ${ }^{34}$, qui, même si elle trouve sa source dans des faits religieux, ne peut pas être ramenée à un trait de réalisme cultuel.

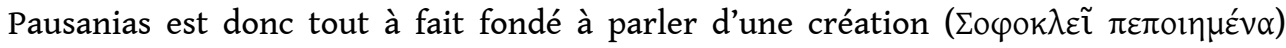
lorsqu'il évoque la version sophocléenne de la mort d'œEdipe ${ }^{35}$.

Indépendamment de la question de son rapport avec un culte rendu à đEdipe en Attique, l'héroïsation d'Ædipe dans $E$ dipe à Colone a été interprétée, plus généralement, à la lumière des réalités du culte héroïque. C'est ce cadre interprétatif que je voudrais mettre à l'épreuve de la tragédie de Sophocle dans le deuxième temps de cet exposé, en m'intéressant successivement à la définition d'€Edipe comme héros, à la représentation de son pouvoir et à ce que le texte laisse entrevoir ou occulte des modalités de sa mort et du lieu de sa sépulture.

\section{L'héroïsation d'F́dipe à la lumière du culte héroïque ${ }^{36}$}

L'héroïsation, au sens religieux du terme, peut être considérée comme la consécration ou le point d'orgue de la destinée extraordinaire du personnage qui en est l'objet. Pour apprécier si et jusqu'à quel point la fin d'ÆEdipe se laisse analyser dans ce cadre, il est légitime de se demander d'abord en quoi la nature et le destin d'œEdipe, tels qu'ils sont représentés dans la tragédie, le désignent comme un candidat possible à l'héroïsation.

\section{1. ㄷdipe ce héros ${ }^{37}$}

Si la première condition pour prétendre au statut héroïque réside dans le caractère exceptionnel, ou extrême de la vie du personnage considéré38, alors nul doute qu'œEdipe est un candidat de choix. De son exposition sur le Cithéron à ses crimes involontaires et à la déchéance ultime de l'exil et de la mendicité, son destin se distingue davantage par la somme des épreuves subies et des souffrances endurées que par des exploits guerriers ou des services rendus à l'humanité, à l'exception notable de la résolution de l'énigme de la Sphinx, libératrice et salvatrice pour la cité de Thèbes ${ }^{39}$. La passion et les excès de son caractère ${ }^{40}$, dont la manifestation la plus éclatante est la malédiction lancée contre ses fils - qu'(Edipe renouvelle dans la pièce en congédiant violemment Polynice (v. 1370-1379) - sont une autre composante de son être héroïque. De fait, comme cela a été souligné, les héros grecs ne sont pas assimilables à des saints chrétiens ${ }^{41}$ et, si certains d'entre eux se caractérisent par leur bienveillance et leur philanthropie, đEdipe n'est pas le seul à faire montre de violence et de dureté, y compris au détriment des siens. E. Kearns suggère comme exemple parallèle l'Eurysthée des Héraclides ${ }^{42}$, qui prédit lui-même, lors de sa confrontation finale avec Alcmène, sa transformation en héros protecteur de l'Attique ${ }^{43}$. Mais il y a une différence notable en ce qui concerne la place de cet élément dans la construction de chacune des deux pièces. Dans la tragédie d'Euripide, la destinée post mortem d'Eurysthée n'est révélée 
qu'à la toute fin, comme une surprise ; dans đEdipe à Colone, l'héroïsation d'œEdipe est annoncée dès le début de la pièce, et c'est vers elle que tend toute la tragédie.

\section{2. ㄷdipe, un mortel}

Un autre point qui ressortit à une définition minimale des héros est le fait qu'ils se distinguent des dieux par leur caractère mortel. Qu'en est-il d'CEdipe? Un grand mystère entoure sa disparition dans la pièce de Sophocle : l'événement est annoncé par des signes divins, il se produit, sur les ordres d'ÆEdipe, en l'absence de tout témoin, à l'exception de Thésée, et il est spécifié que le secret doit être entretenu autour du lieu de sa tombe. Cependant, l'atmosphère de merveilleux qui émane du récit de sa fin ne doit pas conduire à douter qu' $\subset$ dipe meurt bel et bien, dans le secret du bois ${ }^{44}$. De fait, la mort d'ÆEdipe est à l'horizon de la pièce, depuis le début de la tragédie. C'est bien la fin de sa vie que lui a annoncée l'oracle d'Apollon, en lui désignant le bois sacré des Euménides comme lieu de son dernier séjour ${ }^{45}$. Lorsqu'un peu plus tard dans la pièce, Ismène lui fait part de l'arrivée prochaine de Créon et de son intention de se saisir de lui, ÆEdipe se projette, le temps d'une réplique, dans l'image d'un mort enterré aux portes de Thèbes ${ }^{46}$. Et quand il révèle à Thésée le profit que lui vaudra sa présence sur le sol d'Athènes, il précise dans les termes les plus explicites que ce bénéfice, lié à son corps et à sa tombe, se révèlera après sa mort et son enterrement ${ }^{47}$. Enfin, lorsque les signes divins - tonnerre, éclairs - se manifestent, đdipe annonce à ses filles que l'heure de sa fin est venue ${ }^{48}$, avant de communiquer à Thésée les instructions qu'il devra observer après sa mort, pour préserver le secret de sa sépulture (v. 1518-1546). Après quoi, il prend congé de la lumière (v. 1549-1550), évoque son passage vers l'Hadès, sous la conduite des divinités infernales (v. 1547-1548 et 1551-1552) et demande aux siens, en une ultime prière, de garder mémoire de lui «même mort $»^{49}$. Le fait que sa mort soit décrite comme une disparition miraculeuse ${ }^{50}$ voire, si l'on corrige le texte du v. $1583^{51}$, comme le passage à une forme de vie éternelle, traduit l'incompréhension du messager face à un événement auréolé de mystère et ne remet pas en question la perception de la fin d'€Edipe qui s'impose dans le reste de la tragédie. Comme on le voit par ce rapide survol, les références explicites à la mort d'Æđdipe, qui émaillent le texte de la tragédie, sont indissociables de la mention de sa tombe et de son corps définitivement fixé dans le sol de l'Attique. Pour la clarté de l'exposé, je diffère l'analyse plus précise de ces autres données, pour les examiner un peu plus tard, avec la question du culte institué en son honneur.

\section{Le pouvoir d'Édipe après sa mort}

La puissance qui reste attachée à đEdipe après sa mort est sans doute l'élément le plus significatif du point de vue de son assimilation à un héros, au sens proprement religieux du terme. Assez tôt dans la pièce, ๔dipe fait allusion au bénéfice ( $\kappa \varepsilon ́ \rho \delta o \varsigma$,

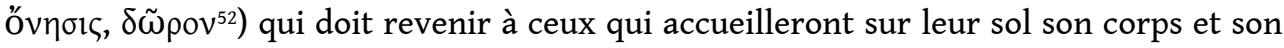
tombeau ${ }^{53}$ et, tout en dévoilant progressivement la signification d'un tel paradoxe, peu crédible au vu de l'état lamentable de sa personne, il l'utilise comme argument à l'égard de ses interlocuteurs successifs pour faire accepter sa présence dans le bois sacré des Euménides, à Colone. Significatif est, à cet égard, l'appel qu'il lance au chœur des vieux Coloniates, avant l'arrivée de Thésée : 


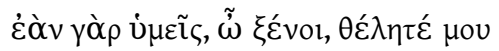

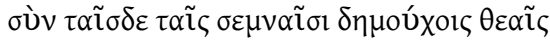

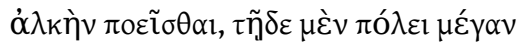

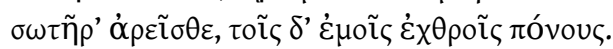

Si vous voulez bien, étrangers,

vous joindre à ces Déesses Redoutables, souveraines de ces lieux,

pour assurer ma défense, vous ménagerez à cette cité un puissant

sauveur, et des revers à mes ennemis. $»^{54}$

Ce passage illustre parfaitement la double nature du pouvoir post mortem d'€Edipe, qui, comme l'avait révélé l'oracle d'Apollon à travers l'antithèse $\kappa \varepsilon ́ \rho \delta \eta / \alpha ̌ \tau \tau \eta^{55}$, est appelé à s'exercer au bénéfice de ses $\mathrm{amis}^{56}$ et au détriment de ses ennemis ${ }^{57}$, conformément au précepte fondamental de la morale archaïque " Helping friends and harming enemies ", dont M. W. Blundell a montré l'importance dans l'éthique sophocléenne ${ }^{58}$. L'idée sousjacente à cette représentation de la puissance du mort est qu'il conserve dans l'au-delà la mémoire des services reçus comme des offenses subies de son vivant, et qu'il est en mesure de répondre aux uns et aux autres par la réaction appropriée, de gratitude ou de colère. Si l'aspect maléfique de ce pouvoir est attesté dans d'autres versions du mythe, son pendant positif, la représentation d'œEdipe comme héros sauveur d'Athènes semble être une innovation de Sophocle, peut-être liée à son désir de célébrer les vertus d'hospitalité de Colone et d'Athènes. Dans le contexte de la pièce, les amis d'ÆEdipe se confondent en effet avec ceux qui l'accueillent, ses ennemis, avec ceux qui l'ont exilé et jeté sur les routes, ce qui recouvre, peu ou prou, l'opposition Athènes/Colone versus Thèbes ${ }^{59}$.

La connaissance que des oracles lui ont donnée du pouvoir qui s'attache à la personne d'CEdipe et à sa tombe est précisément ce qui pousse Créon, le roi de Thèbes, à vouloir s'emparer de lui ${ }^{60}$. Son attitude agressive, qui vise seulement à s'assurer le contrôle de sa personne ${ }^{61}$ et à garantir à sa cité les succès escomptés, sert surtout, dans la pièce, de faire-valoir à l'hospitalité désintéressée de Thésée. La place que Créon entend réserver à la tombe d'€Edipe, à l'extérieur de la cité, aux confins du territoire ${ }^{62}$, et son refus de couvrir son corps de poussière ${ }^{63}$ montrent assez qu'aux yeux des Thébains, ÆEdipe reste un criminel souillé, ce qui permet là aussi de célébrer par contraste la générosité et l'humanité du roi d'Athènes. Au-delà de cette caractérisation différenciée de Thèbes et d'Athènes, à travers l'attitude opposée de leurs rois, la lutte que se livrent les deux cités pour accueillir la tombe d'œdipe est révélatrice du fait que son héroïsation fait sortir Edipe de Thèbes et du cercle familial pour le lier définitivement à la cité d'Athènes ; ce passage du plan de l'oikos à celui de la polis, qui vérifie aussi l'une des caractéristiques des cultes héroïques ${ }^{64}$, est pleinement consacré un peu plus tard par les modalités de la disparition d'ÆCdipe ${ }^{65}$, et par la perception différente que Thésée et les filles d'œEdipe ont de la mort d'CEdipe ${ }^{66}$.

\section{Un allié dans les guerres entre Athènes et Thèbes}

Si la possession de ce pouvoir post mortem tend donc à qualifier @Edipe comme héros, au sens religieux du terme, le domaine dans lequel il est censé s'exercer, qui est celui des entreprises militaires et des batailles à venir entre Athènes et Thèbes (cf. v. 602-623), concorde lui aussi avec certaines représentations traditionnellement attachées aux héros. L'acharnement de Créon à vouloir se saisir d'ÆEdipe est expressément lié à l'idée

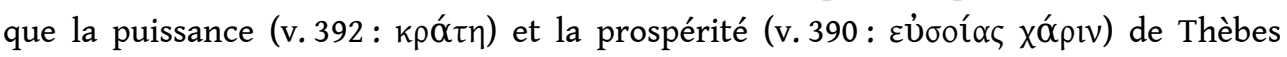


dépendent de la présence de sa tombe sur le territoire de la citée ${ }^{67}$. Edipe ne s'y trompe pas, qui, lorsqu'il démasque les prétextes sous lesquels Créon tente de dissimuler ses véritables intentions, l'accuse de vouloir se servir de lui pour mettre sa cité à l'abri des attaques d'Athènes ${ }^{68}$. Ce calcul, vicié par l'occultation des fondements éthiques sur lesquels reposent les relations entre morts et vivants, se heurte à la colère (v. 411 : ópүñऽ) présente et future d'ÆEdipe, qui condamne par avance à l'échec toutes les entreprises thébaines. La cité d'Athènes se voit en revanche offrir, en échange de l'accueil bienveillant qu'elle lui a réservé, et à la condition expresse de tenir secret le lieu de sa sépulture, la protection toute-puissante du mort et de son tombeau, «aide égale à mille boucliers, voire à une armée de renfort accourue d'un pays voisin " $"$. À certains égards, la victoire remportée par les troupes de Thésée sur celles de Créon, qui permet au roi d'Athènes de remettre entre les mains d'ÆEdipe ses filles précédemment ravies par l'agresseur thébain, préfigure les succès à venir d'Athènes dans les guerres contre Thèbes. Elle fait peut-être aussi écho, dans le temps de la représentation, à l'histoire récente d'Athènes et à la victoire remportée en 408/407 par les Athéniens sur la cavalerie thébaine engagée aux côtés du roi Agis, à l'occasion d'un coup de force des Spartiates dans la région de Colone $^{70}$. Un lien supplémentaire entre le mythe et l'histoire est tissé par une scholie au discours Sur les quatre d'Aelius Aristide ${ }^{71}$, qui rapporte que lors de cet affrontement, Ædipe serait apparu sur le champ de bataille, aux côtés des Athéniens, leur assurant ainsi la victoire. C'est là, comme on le sait, un épisode ordinaire - et presque attendu - dans les vies héroïques (que l'on songe, par exemple, à l'apparition de Thésée, lors de la bataille de Marathon : Plut., Thésée, 35, 6-7) et le signe d'une forme de consécration d'ÆEdipe comme héros. On voit comment la tragédie de Sophocle, en construisant la représentation d'cEdipe comme puissance protectrice d'Athènes, a pu favoriser son intégration à la mythologie héroïque commune, même si la signification du parcours tragique d'ÆEdipe va au-delà de ces rencontres avec le modèle héroïque.

\section{La tombe d'Édipe}

Les interprètes portés à voir, dans le scénario imaginé par Sophocle pour la fin d'Ædipe, une projection des réalités religieuses peuvent y trouver d'autres éléments conformes aux realia du culte héroïque. Le plus évident est l'importance accordée à la tombe d'CEdipe tout au long de la tragédie. Dans l'imaginaire mythologique, comme dans la réalité de la pratique religieuse, la tombe, que le héros est censé habiter (même s'il est souvent représenté, en même temps, comme un hôte de l'Hadès) est en effet le point de contact entre les vivants et les morts, le lieu mémoriel spécifiquement dédié au souvenir du défunt et le réceptacle des offrandes qui lui sont adressées dans le cadre du culte dont il est l'objet. Le caractère local, topique du culte héroïque, qui est régulièrement noté par les historiens de la religion grecque, résulte directement du fait que la tombe en est le centre. Or, l'importance et l'unicité du lieu qui doit abriter le tombeau d'CEdipe sont soulignées à l'envi par la tragédie de Sophocle. Les détails topographiques donnés par le messager qui fait le récit de sa disparition mystérieuse (v. 1590-159772), qu'elles renvoient à une géographie réelle connue des spectateurs, comme le suggère le scholiaste, ou se laissent plutôt interpréter comme les éléments d'une géographie symbolique ${ }^{73}$, comme je le crois plus volontiers, identifient très précisément l'emplacement de la mort d'ÆEdipe, en l'ancrant dans le paysage de l'Attique. L'idée, soulignée par les tentatives d'appropriation de Créon, selon laquelle la 
protection d'CEdipe doit s'exercer en faveur des habitants du pays abritant sa tombe est elle aussi conforme aux croyances communes relatives au pouvoir des héros.

\section{Le secret autour de la sépulture}

29 Le secret dans lequel doit être tenu le lieu de sa tombe est une donnée plus difficile à appréhender dans le cadre du culte héroïque ${ }^{74}$. ๔dipe fait de ce secret, dont il n'excepte que Thésée et ses successeurs, la condition ultime de l'aide qu'il s'engage à apporter à la cité d'Athènes. Cela ressort clairement des instructions qu'il donne à Thésée, lorsqu'après la manifestation des signes annonciateurs de sa mort, il lui révèle la nature du don qu'il lui a promis :

Je vais t'apprendre, fils d'Égée, de quels biens

exempts de la vieillesse et de ses peines, vous allez disposer, toi et la cité.

Le lieu où je dois mourir, je vais moi-même t'y conduire

sur-le-champ, sans toucher aucun guide.

Ce lieu, ne révèle à aucun homme

ni où il se cache, ni dans quels parages il se trouve,

de manière à ce qu'il te procure une protection égale à mille boucliers,

à la lance secourable d'un peuple voisin.

Les choses interdites que la parole ne met pas en branle,

tu les apprendras toi-même quand tu iras là-bas, toi seul.

Car je ne saurais les dévoiler ni à l'un des citoyens d'ici,

ni à mes enfants, malgré l'affection que je leur porte.

Garde-les pour toi, et quand tu seras arrivé

au terme de ta vie, fais-les savoir seulement

à ton héritier, et qu'il les révèle à son successeur, et ainsi de suite.

Ainsi la cité que tu habites restera à l'abri des ravages

que pourrait lui infliger la race des Spartes. ${ }^{75}$

À travers ces directives, ๔Edipe s'institue clairement en héros protecteur d'Athènes, mais selon un scénario tout à fait original, dont l'élément central est le secret. Ce secret apparaît comme une sorte de privilège royal : seul (cf. póvoc, v. 1527 et 1531) le roi du pays en est le dépositaire, à chaque génération, alors que de façon non moins remarquable, en sont exclus aussi bien les filles d'ÆEdipe que les gens du pays, représentés ici par le chœur. L'orchestration, par đedipe lui-même, de sa sortie finale et de ses derniers instants établit les conditions concrètes de l'instauration de ce secret. Antigone, Ismène et le serviteur qui fera ultérieurement le récit de ce qu'il a vu à distance, l'accompagnent jusqu'à un certain point, mais ils sont sommés ensuite de faire demi-tour (v.1638-1643). Seul Thésée suit đEdipe jusqu'au lieu de sa tombe et assiste à ses derniers instants (v. 1643-1644, 1650-1655).

Le texte est peu explicite sur les raisons de ce secret. Les commentateurs supposent, en général, qu'il est lié à la nécessité de protéger la tombe d'ÆEdipe des entreprises de l'ennemi $^{76}$, et invoquent à l'appui de cette hypothèse quelques - rares - exemples parallèles, principalement, celui des filles d'Érechthée, à Athènes, dont le souvenir est préservé par un fragment de l'Érechthée d'Euripide ${ }^{77}$, et celui de Dircè, à Thèbes, que Plutarque cite dans un passage de son traité Sur le démon de Socrate ${ }^{78}$ (cf. aussi les tombes secrètes de Nélée et de Sisyphe à l'Isthme de Corinthe, dont parle Pausanias ${ }^{79}$ ). Le premier exemple est en réalité assez peu comparable à celui d'Æđdipe, d'une part parce qu'il n'y est pas expressément question de secret ${ }^{80}$, d'autre part parce que l'accent y est mis surtout sur le culte et les rites (sacrifices sanglants et libations sans vin) institués par la déesse Athéna en l'honneur des filles d'Érechthée, enfin, parce que les filles 
d'Érechthée sont, dans une combinaison originale de culte héroïque et de catastérisme, élevées au rang de divinités, sous le nom de Hyacinthides. Le cas de Dircè parait plus proche ${ }^{81}$, dans la mesure où l'emplacement de la tombe de Dircè est destiné à rester

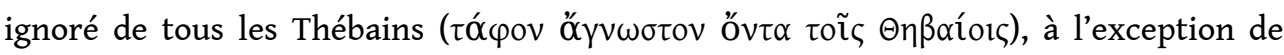
l'hipparque, qui le transmet à son successeur à sa sortie de charge. Comme dans EEdipe à Colone, donc, le secret de la tombe est un privilège associé à l'exercice du pouvoir (en l'occurrence ici, le pouvoir militaire), mais aussi à une sorte de refondation symbolique de la cité autour de l'une de ses figures tutélaires. La différence avec Edipe tient cependant au fait que la transmission du secret et du pouvoir d'un hipparque à son successeur s'accompagne de rites nocturnes accomplis sur la tombe.

\section{Anomalies : absence de rites et absence de tombe ?}

32 Rien de tel n'est mentionné dans le cas d'CEdipe, et il n'est dit nulle part dans la tragédie que sa tombe devient le lieu d'un culte, exceptionnel ou régulier ${ }^{82}$. L'idée même d'un tel culte est en contradiction avec celle de secret, et c'est là que la représentation de l'héroïsation d'ÆEdipe prend le plus clairement ses distances avec le modèle du culte héroïque. Du fait même de ce secret, ce n'est pas autour de la tombe que se construit ici le rapport entre les vivants et le mort. Non que ce rapport soit totalement oublié : de façon significative, la dernière parole qu'œEdipe adresse aux siens

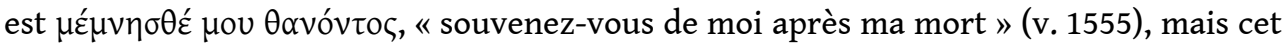
appel à la mémoire des vivants n'en fait que mieux ressortir le fait que la tombe d'Æ̇dipe n'assure pas la fonction mémorielle normalement dévolue aux édifices funéraires. De façon tout à fait cohérente, elle n'est d'ailleurs jamais désignée dans la

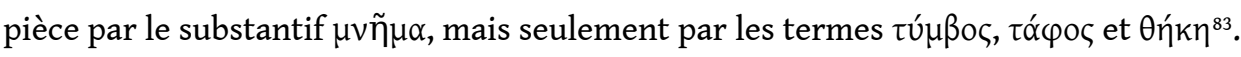

Le secret dont elle est entourée n'est pas le seul élément remarquable dans la représentation de la tombe d'Ædipe. Un autre trait notable, et moins souvent commenté, est le fait que la tombe semble préexister à la mort du héros. Depuis le début de la pièce, l'accent a été mis sur le lieu, situé à proximité, ou peut-être dans le sanctuaire des Euménides, que l'oracle d'Apollon lui a désigné comme son dernier séjour (v. 89-92). C'est vers ce lieu - $\chi \tilde{\omega} \rho$ ov, v. 1520 et 1540, en tête de vers dans les deux cas - qu'ÆEdipe commence à se diriger, sous la conduite des divinités infernales, lorsqu'il perçoit les signes annonciateurs de sa mort. Mais dans les instructions qu'il donne à ses filles pour les inviter à se joindre au cortège, ÆEdipe évoque aussi la « tombe sacrée $»^{84}$ dans laquelle il doit être enseveli.

Dans son commentaire, Guidorizzi suggère que le mot $\tau u ́ \mu \beta o \varsigma$ doit être entendu dans ce passage comme renvoyant non pas au sépulcre à proprement parler, mais au lieu de sépulture d'œEdipe ${ }^{85}$. Mais ce lieu, nous l'avons vu, est désigné dans la tirade d'œEdipe

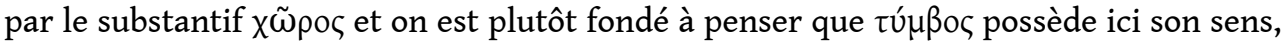
habituel à l'époque classique ${ }^{86}$, de "tombeau ", même si la mention de cette "tombe sacrée » ne s'accompagne d'aucun élément de description permettant d'en imaginer la forme ou l'aspect. Si l'on accepte cette lecture, qui s'ancre dans les usages du grec, l'écart se creuse encore avec les procédures d'instauration du culte héroïque: le tombeau d'œdipe n'est pas érigé au moment de sa mort, pour recevoir sa dépouille et permettre la célébration du rite funèbre, à la différence de ce que l'on trouve, par exemple, à la fin de l'Ajax ${ }^{87}$. CEdipe rejoint, avec l'accord et sous l'égide des dieux, le lieu et le tombeau qui lui ont été assignés par le destin, et il accède, ce faisant, à un statut 
supérieur à celui de l'humanité ordinaire, que figure déjà, dans le temps du drame, la métamorphose qui prélude à sa sortie finale. Le contraste est en effet total entre l'image du vieux mendiant aveugle, incapable d'assurer son pas sans le soutien d'Antigone, que l'on voit arriver à Colone, au début de la tragédie, et celle du héros régénéré qui prend la tête du cortège pour gagner le lieu de son dernier séjour et orchestrer, chemin faisant, les étapes du scénario qui culmine dans sa disparition miraculeuse. Ce contraste n'est pas moins frappant du point de vue du statut religieux d'ÆEdipe : le criminel que les Coloniates voulaient chasser par crainte de la souillure dont il était porteur (cf. v. 226-236) accède, au moment où il va mourir, à une forme de sacralité, qui le place à part des autres hommes et le rend intouchable (v. $1544: \mu \grave{\eta}$ $\psi \alpha u ́ \varepsilon \tau ')$. Du rejet de l'être souillé à l'inviolabilité de l'être sacré, l'interdit du contact est ainsi resémantisé en un sens positif qui résume à lui seul le renversement opéré par la tragédie.

On voit que la représentation de ce parcours singulier, qui se construit dès le début de la pièce, avec l'arrivée d'œEdipe dans le bois sacré des Euménides, déborde - même si elle les utilise - les catégories et le cadre rituel du culte héroïque. Significative est aussi, à cet égard, l'ambiguïté dont le texte est porteur, en ce qui concerne l'ensevelissement

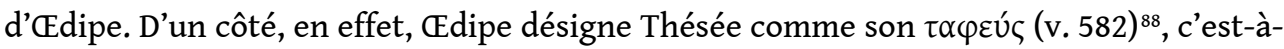
dire, avec la valeur du suffixe des noms d'agent, son «fossoyeur ", celui qui est chargé de le mettre dans la tombe ; mais d'un autre côté, Ismène oppose au désir exprimé par Antigone de voir la tombe de son père ${ }^{89}$ le fait qu'il est ö $\tau \alpha \varphi \circ \varsigma$, « privé de sépulture ${ }^{90}$. Je ne crois pas, là non plus, que la contradiction puisse être résolue en supposant simplement, avec Kamerbeek ${ }^{91}$, que l'adjectif signifie dans ce passage "privé de rituel funèbre ", plutôt que " privé de tombe ». Avec ce sens, en effet, le lien logique entre la demande d'Antigone et la réplique de sa sœur n'apparaîtrait pas. D'autre part, dans ses autres occurrences, l'adjectif s'applique toujours à un mort qui n'a pas encore reçu de tombe, qui n'a pas été enterré, ou à qui on refuse l'octroi d'une sépulture, comme dans le cas, fameux entre tous, de Polynice ${ }^{92}$, dont le poète entend peut-être évoquer l'image dans l'esprit des spectateurs, en employant le qualificatif à propos de son père. La contradiction apparente entre les deux passages s'explique en réalité par le dispositif unique qui fait de Thésée le seul témoin des derniers instants d'€Edipe (il est tombé, dit

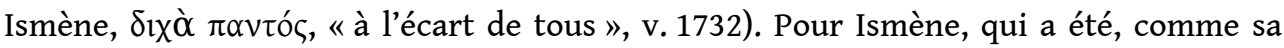
sœur, privée du rôle normalement dévolu aux proches du défunt - la possibilité de pleurer le mort et de déposer des offrandes sur sa tombe -, tout se passe comme si CEdipe n'avait pas de tombe ${ }^{93}$. Et de fait, l'incertitude ainsi entretenue sur l'existence d'une véritable tombe destinée à accueillir le mort reste en partie irrésolue par le mystère qui entoure les derniers instants d'ÆEdipe. Le messager qui, de l'endroit où il a été contraint de s'arrêter, n'a eu qu'une perception lointaine de l'événement ${ }^{94}$, se demande même si ce n'est pas la terre qui s'est ouverte pour permettre à đEdipe de passer chez les morts ${ }^{95}$. Cette narration à distance, qui échappe aussi aux conventions $\mathrm{du}$ récit de messager, laisse le spectateur dans l'ignorance de ce qu'il advient du corps d'œEdipe, des modalités de sa disparition, et du statut auquel il accède par ce passage dans l'autre monde.

Ces quelques réflexions auront montré, je l'espère, que la représentation de la fin d'ÆEdipe dans $\Subset$ dipe à Colone est partiellement (mais seulement partiellement) informée par le modèle du culte héroïque, et que le poète fait fond sur l'expérience religieuse des spectateurs pour les impliquer intellectuellement et émotionnellement dans la 
métamorphose de son héros. Les incertitudes de la chronologie font qu'il est plus difficile d'apprécier l'influence que les cultes rendus à đEdipe en Attique peuvent avoir exercée sur le choix et le traitement, par Sophocle, de cette matière mythique. Mais il en va de ces données cultuelles comme des traditions mythologiques antérieures : elles ne représentent, au mieux, qu'un matériau que le poète a travaillé et façonné en une composition originale, dont il y a lieu de penser qu'elle a elle-même laissé son empreinte sur les récits et sur les cultes ultérieurs. Si donc les realia religieux, qui constituent un univers de référence commun au poète et aux spectateurs, ne doivent pas être négligés dans la lecture de la pièce, il n'en reste pas moins que l'héroïsation d'ÆEdipe est avant tout un événement dramatique, l'aboutissement d'un parcours tragique qui voit le vieil aveugle proscrit transformé en une puissance protectrice d'Athènes. L'impression qui domine le récit de sa disparition est celle d'un thauma inaccessible à l'entendement humain, et les références ponctuelles aux données du culte héroïque ne semblent, en définitive, être insérées dans le texte que pour souligner à quel point la mort d'CEdipe est un événement irréductible à tout modèle. C'est finalement beaucoup moins l'instauration d'un culte que l'accomplissement d'un destin singulier qui est ainsi donné à voir.

\section{BIBLIOGRAPHIE}

AмIEсH 2007 : Ch. Amiech, « Sur l'authenticité du dénouement », in J. Alaux (éd.), Les

Phéniciennes. La famille d'Édipe entre mythe et politique, Paris 2007, 111-128.

AUSTIN 1967 : C. Austin, « De nouveaux fragments de l'Érechthée d'Euripide », in Recherches de papyrologie IV, Paris 1967, 11-67 et pl. I-II.

BERNARD 2001 : W. Bernard, Das Ende des Ödipus bei Sophokles. Untersuchung zur Interpretation des 'Ödipus auf Kolonos', Zetemata 107, München 2001.

BLUNDELL 1989 : M. W. Blundell, Helping Friends and Harming Enemies, Cambridge 1989.

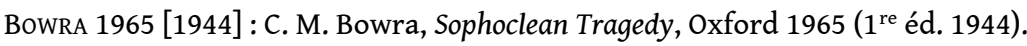

BRELICH 1958 : A. Brelich, Gli eroi greci. Un problema storico-religioso, Roma 1958.

BURIAN 1972 : P. Burian, «Supplication and hero cult in Sophocles' Ajax », Greek, Roman, and Byzantine Studies 13 (1972), 151-156.

BURIAN 1974 : P. Burian, « Suppliant and Savior: EEdipus at Colonus », Phoenix 28, 4 (1974), 408-429.

BURKERT 1985 : W. Burkert, Greek Religion (éd. or. Stuttgart 1977), trad. angl., Blackwell Publishing 1985.

CALAME 1998 : C. Calame, « Mort héroïque et culte à mystère dans l'ÆEdipe à Colone de Sophocle », in F. Graf (hrsg.), Ansichten griechischer Rituale. Geburstags-Symposium für Walter Burkert, Stuttgart 1998, 326-356.

CHANTRAINE 1968 : P. Chantraine, Dictionnaire étymologique de la langue grecque, Paris 1968. 
CURRIE 2012 : B. Currie, « Sophocles and Hero Cult », in K. Ormand (ed.), A Companion to Sophocles, Wiley-Blackwell 2012, 331-348.

EASTERLING 2006 : P. Easterling, " The Death of @Edipus and what happened next », in D. Cairns, V. Liapis (eds.), Dionysalexandros. Essays on Aeschylus and his Fellow Tragedians in Honour of A. F. Garvie, Swansea 2006, 133-150.

EDMUNDS 1981 : L. Edmunds, "The Cults and the Legend of CEdipus ", Harvard Studies in Classical Philology 8 (1981), 221-238.

EDMUNDS 1996 : L. Edmunds, Theatrical Space and Historical Place in Sophocles' Edipus at Colonus, London 1996.

EКROTH 2002 : G. Ekroth, The sacrificial rituals of Greek hero-cults in the Archaic to the early Hellenistic periods, Kernos, supplement 12, Liège 2002.

EKROTH 2007 : G. Ekroth, « Heroes and hero-cult » in D. Ogden (ed.), A Companion to Greek Religion, Wiley-Blackwell 2007, 100-114.

FARNELL 1921 : L. Farnell, Greek Hero Cults and Ideas of Immortality, Oxford 1921.

FERRARI 2003 : F. Ferrari, « Edipo a Colono 1583: critica del testo e critica storico-religiosa ", in G. Avezzù (a cura di), Il dramma sofocleo: testo, lingua, interpretazione, Drama 13, Stuttgart-Weimar 2003, 125-142.

FESTUGIÈRE 1973 : A.-J. Festugière, "Tragédie et tombes sacrées ", Revue de l'histoire des religions 184, 1 (1973), 3-24.

FOWLER 1999 : R. L. Fowler, « Three Places of the Trachiniae », in J. Griffin (ed.), Sophocles Revisited. Essays Presented to Sir Hugh Lloyd-Jones, Oxford 1999, 161-175.

Guidorizzi 2008 : G. Avezzù, G. Guidorizzi, Sofocle. Edipo a Colono, Roma 2008.

HENRICHS 1983 : A. Henrichs, "The ‘Sobriety’ of CEdipus: Sophocles OC 100 Misunderstood », Harvard Studies in Classical Philology 10 (1983), 87-100.

HENRICHS 1993 : A. Henrichs, "The Tomb of Aias and the Prospect of Hero Cult in Sophocles », Classical Antiquity 12 (1993), 165-180.

JEBB 1900 : R. C. Jebb, Sophocles. The Plays and Fragments, Part II. CEdipus Coloneus, Cambridge 1900. JoUANNA 2007 : J. Jouanna, Sophocle, Paris 2007

KAMERBEEK 1984 : J. C. Kamerbeek, The Plays of Sophocles, Part VII. The CEdipus Coloneus, Leiden 1984. KEARNS 1989 : E. Kearns, The Heroes of Attica, Bulletin of the Institute of Classical Studies, suppl. 57, London 1989.

KeLly 2009 : A. Kelly, Sophocles: Edipus at Colonus, Duckworth 2009.

KowALzIG 2006 : B. Kowalzig, "The Aetiology of Empire? Hero-cult and Athenian Tragedy », in J. Davidson, F. Muecke, P. Wilson (eds), Greek Drama III. Essays in Honour of Kevin Lee, Bulletin of the Institute of Classical Studies, Suppl. 87, London 2006, 79-98.

LACORE 1995-1996: M. Lacore, « Mort et divinisation des filles du roi d'Athènes dans l'Erechthée d'Euripide », Kentron 11, 2-12, 1 (1995-1996), 89-107.

LINFORTH 1951 : I. M. Linforth, « Religion and Drama in the 'ÆEdipus at Colonus' », University of California Publications in Classical Philology 14 (1951), 75-192. 
LLOYD-JONES, Wilson 1990 : H. Lloyd-Jones, N. Wilson, Sophoclea. Studies on the Text of Sophocles, Oxford 1990.

MARKANTONATOS 2002 : A. Markantonatos, Tragic Narrative. A Narratological Study of Sophocles' Edipus at Colonus, Berlin-New York 2002.

MARKANTONATOS 2007 : A. Markantonatos, CEdipus at Colonus. Sophocles, Athens, and the World, Berlin 2007.

MASTRONARDE 1994 : D. J. Mastronarde, Euripides, Phoenissae, edited with introduction and commentary, Cambridge 1994.

MÉAUTIS 1940 : G. Méautis, L'đEdipe à Colone et le culte des héros, Recueil de la Faculté des Lettres de Neuchâtel 19, Neuchâtel 1940.

MiKALSON 1991 : J. D. Mikalson, Honor Thy Gods. Popular Religion in Greek Tragedy, Chapell HillLondon 1991.

PARKER 2011 : R. Parker, On Greek Religion, Cornell Publishing 2011.

ROSENMEYER 1952 : T. Rosenmeyer, « The Wrath of @dipus », Phoenix 6 (1952), 92-112.

RUDHARDT 1983 : J. Rudhart, « Edipe et les chevaux », Museum Helveticum 40 (1983), 131-139.

RUDHARDT 1992 [1958] : J. Rudhardt, Notions fondamentales de la pensée religieuse et actes constitutifs du culte dans la Grèce classique, Paris 1992 (1 ${ }^{\text {re }}$ éd. 1958).

SEAFORD 1994a : R. Seaford, Reciprocity and Ritual, Oxford 1994.

SEAFORD 1994b : R. Seaford, « Sophocles and the mysteries », Hermes 122 (1994), 275-288.

SEGAL 1981 : C. Segal, Tragedy and Civilization. An Interpretation of Sophocles, Cambridge, MA 1981.

TILG 2004 : S. Tilg, « Die Symbolik chthonischer Götter in Sophokles’ Ödipus auf Kolonos », Mnemosyne 57 (2004), 407-420.

VIAN 1963 : F. Vian, Les origines de Thèbes, Paris 1963.

WiLSON 1997 : J. Wilson, The Hero and the City. An Interpretation of Sophocles' Ædipus at Colonus, Ann Arbor 1997.

WINNINGTON-INGRAM 1980 : R. P. Winnington-Ingram, Sophocles. An Interpretation, Cambridge 1980.

\section{NOTES}

1. L'un des premiers à l'avoir souligné est BowRA 1965 [1944], 307-355.

2. Voir la synthèse de CURRIE 2012.

3. Sophocle, Ajax, 1171-1181.

4. BURIAN 1972 ; HENRICHS 1993 ; CURRIE 2012, 333-336.

5. FOWLER 1999 ; CURRIE 2012, 336-337.

6. Médée, Les Héraclides, Hippolyte, Iphigénie en Tauride.

7. Comme le souligne à juste titre EASTERLING 2006.

8. Voir par ex. SEAFORD 1994a, 130 qui tend à lire la tragédie de Sophocle comme une étiologie dramatisée du culte d'ÆEdipe à Athènes.

9. EASTERLING 2006. Parmi les interprétations plus anciennes, celle de BURIAN 1974, qui lit Édipe à Colone à la lumière du schéma dramatique des tragédies de la supplication, laisse également de côté la question des emprunts de Sophocle aux réalités cultuelles. 
10. Sur les traditions relatives à la mort d'ÆEdipe et à ses cultes, voir F ARNELL 1921, 332-334 ; EDMUNDS 1981 ; KEARNS 1989, 208-209 ; EDMUNDS 1996, 95-100.

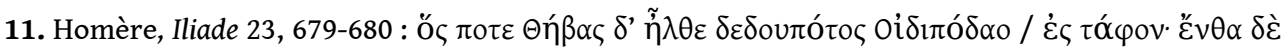

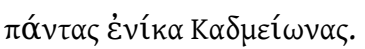

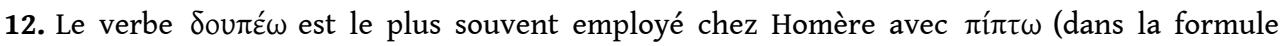

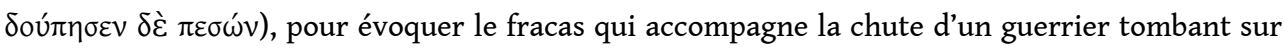
le champ de bataille. Voir Il. 4, $504 ; 5,42,540,617$, etc.

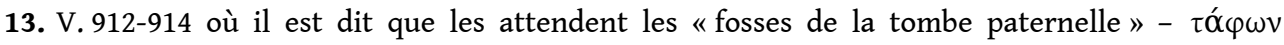

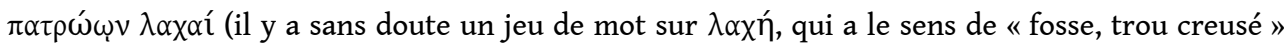

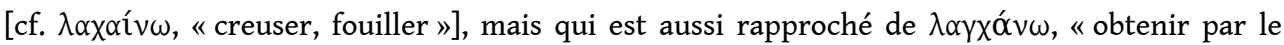
sort » [ce qu'ils obtiennent par le sort, c'est une part de la tombe paternelle]; cela ne signifie pas nécessairement qu'ils vont être enterrés dans la même tombe que leur père); v. $1004: \pi \tilde{\eta} \mu \alpha$

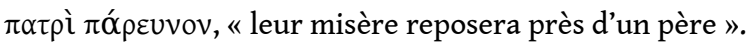

14. Sophocle, Antigone, 897-902.

15. FGrH $382 \mathrm{~F} 2$ (scholie à Sophocle, OC 91 : commentaire à l'oracle d'Apollon qui annonce à đEdipe sa mort à Colone).

16. Et des vers 1703-1707 des Phéniciennes d'Euripide, qui annoncent l'exil d'ÆEdipe et sa mort à Colone. Je laisse de côté ces vers, dont l'authenticité ne fait pas l'objet d'un consensus dans la critique et que certains considèrent comme un ajout postérieur, influencé par $\mathbb{E}$ dipe à Colone. Sur la question de l'authenticité de la fin des Phéniciennes, voir АмІесн 2007 (qui défend l'attribution à Euripide de l'ensemble du dénouement, tout en reconnaissant [122, n. 29] que « les v. 1703-1707 sont sujets à caution", notamment parce qu'ils entrent en contradiction avec le v. 1736, où Antigone désigne par l'adverbe indéfini nou le lieu de la mort d'œdipe). L'authenticité de ces vers est également défendue par MASTRONARDE 1994 ad loc.; MARKANTONATOS 2007, 57 ; elle est mise en doute par KEARNS, 1989, 208 (avec renvoi à la bibliographie antérieure).

17. Androtion d'Athènes (IV s. av. J.-C.), FGrH 324 F62 (scholie V Hom. Od. 11, 271).

18. EDMUNDS 1996, 95-96 suppose, sur la base des différences que son récit présente par rapport à la tragédie de Sophocle, qu'Androtion représente une tradition parallèle et indépendante.

19. Pausanias, 1, 28, 6-7.

20. RUDHARDT 1983, 137 n. 65, suggère que cet effort de conciliation entre tradition homérique et tradition attique repose sur l'idée peut-être erronée selon laquelle les monuments consacrés à ๔dipe en Attique passaient pour abriter sa dépouille. Le secret qui entoure la disparition d'œ®dipe dans la pièce de Sophocle pourrait trouver une explication si tel n'était pas le cas.

21. Ce transfert des ossements d'œEdipe fait songer à celui des os de Thésée de Skiros à Athènes, ordonné par Cimon, au V $\mathrm{V}^{\mathrm{e}}$ s. av. J.-C.

22. Pausanias, $1,30,4$.

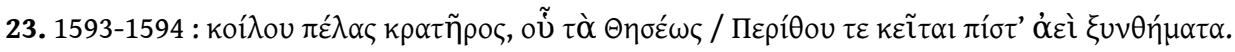

24. Comme le laisse penser le fonctionnement de la coordination, qui distingue deux couples: Pirithous/Thésée d'une part, ๔edipe/Adraste d'autre part.

25. Notamment ROSENMEYER 1952, 98-100.

26. KAMERBEEK 1984, introduction 2-3, qui souligne que l'ancrage marqué dans le contexte local, avec la mention de lieux très précis, trahit des souvenirs personnels de Sophocle : « He doubtless borrowed this subject-matter from local legend connected with the shrine of the Erinyes at Colonus, his

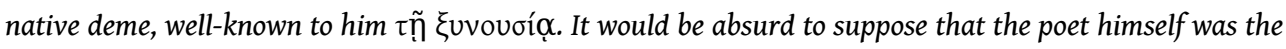
originator of ¿Edipus' connection with Colonus ; a hero-cult existing in his time somewhere in the precinct of the Eumenides and its aetiological myth must have been at the bottom of the dramatized fiction for the latter to be at all credible to Sophocles' audience, who where well-acquainted with the locality, its cults and legends. The intensity in the handling of locality and 'ambiance' is surely to be ascribed to the personal feelings and memories of the poet himself $»$. 
27. JOUANNA 2007, 144 : «Sophocle pouvait difficilement revendiquer pour le dème où il était né la tombe d'ÆEdipe en entourant d'un certain mystère l'endroit exact où elle se trouvait si les spectateurs savaient qu'elle se trouvait à Athènes, dans le sanctuaire des Érinyes, non loin du théâtre de Dionysos ... Dès lors, la tombe que l'on a montrée à Pausanias dans le sanctuaire des Érinyes à l'intérieur des murs doit être une création plus récente, probablement à la faveur d'une assimilation entre deux sanctuaires consacrés aux mêmes déesses, le sanctuaire urbain d'Athènes et le sanctuaire campagnard de Colone ".

28. Dans l'appendice qu'elle a consacré à cette question, KEARNS 1989, 208-209 part du principe qu'il n'a pas pu exister deux traditions contemporaines indépendantes, plaçant, pour l'une, la tombe d'ÆEdipe à Colone, et l'autre, sur l'Aréopage. Sans se prononcer très fermement, elle fait valoir qu'il y a trop de témoignages qui lient đEdipe à Colone pour supposer que tout vient de Sophocle; elle cite en particulier une scholie au v. 57 d'OC, qui fait état d'une attaque des Béotiens contre Colone (mais ne mentionne pas $(\mathbb{d i p e}$ ), et qui fournit ainsi un témoignage pour un conflit entre Athènes et Thèbes dans la région de Colone.

29. Commentaire à $1522 \mathrm{f}$. : "note how Soph. uses the vagueness of the local legend as to the grave $»$.

30. KeLLy 2009, 43-44: «The playwright... manages to leave room for something of both cults or traditions ".

31. Cf. v. 62-3 où le Coloniate qui instruit @edipe sur le caractère sacré du lieu et les cultes qui y

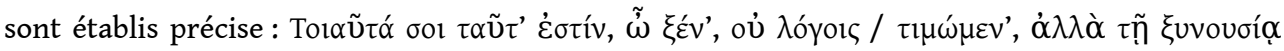
$\pi \lambda \varepsilon ́ o v$, « ce sont là, étrangers, des choses que l'on n'honore pas dans des histoires, mais plutôt en fréquentant les lieux ».

32. Cf. en particulier le premier stasimon, qui est un éloge conjoint de Colone et d'Athènes.

33. Sur l'absence de rites et sur le secret qui entoure le lieu de sépulture d'Ædipe dans la tragédie de Sophocle, voir plus loin.

34. Sur ce point, voir notamment TILG 2004.

35. Cette expression peut s'interpréter à la lumière de ce qu'Aristote dit de la poésie, au c. 9 de la Poétique: "Tout cela montre à l'évidence que le poète doit être un fabricant d'histoires plutôt qu'un fabricant de vers, d'autant qu'il est poète en vertu de la représentation, et qu'il représente des actions. Et quand bien même il représente, à l'occasion, des événements qui sont réellement arrivés, il n'en est pas moins poète ".

36. Pour la bibliographie d'ensemble sur les cultes héroïques, voir l'introduction à ce volume. Le développement suivant se fonde essentiellement, pour la définition de la catégorie des héros et les traits distinctifs du culte héroïque, sur BRELICH 1958 ; RUDHARDT 1992 [1958], 127-135 ; BURKERT 1985, 203-208 ; EKROTH 2002 ; EKROTH 2007 ; PARKER 2011, 103-123.

37. Sur la caractérisation d'€Edipe comme héros et sur la signification de son héroïsation dans la pièce, voir notamment MIKALSON 1991, 39-41; W ILSON 1997, 167-186; B ERNARD 2001, 43-58; MARKANTONATOS 2007, 140-156 ; CURRIE 2012, 339-341.

38. ЕкRотн 2007, 104 : « Having been extreme in some sense, in life or death, was the primary reason for heroic status ».

39. Cf. WILSON 1997, 178, qui souligne (n. 17) qu'ÆEdipe est plus guerrier dans certaines versions antérieures à Sophocle.

40. Sur cette caractérisation d'€Edipe dans Edipe à Colone, voir MÉAUTIS 1940, 42-56 ; WINNINGTONINGRAM 1980, 257-260.

41. Au sens où ils ne sont pas nécessairement bienveillants envers les hommes de leur vivant, ni caractérisés par leurs vertus (cf. PARKER 2011, 104-105). L'héroïsation ne peut donc pas se laisser analyser systématiquement comme une « récompense ». FESTUGIÈRE 1973 souligne cependant les similitudes existant entre la représentation de la mort et du tombeau d'ÆEdipe dans $\Subset$ dipe à Colone et les traditions relatives à certains saints chrétiens. 
42. KEARNS 1989, 50 : «both heroes are foreigners, unlikely heroes for Athens, and furthermore one is an enemy, the other a blind beggar never purified from his horrendous if involuntary deeds ». Sur le parallèle entre CEdipe et Eurysthée, voir aussi FESTUGIÈRE 1973, 22-24.

43. Héraclides, v. 1030-1037.

44. Sur ce point, voir BLUNDELL 1989, 255 ; EASTERLING 2006, 138.

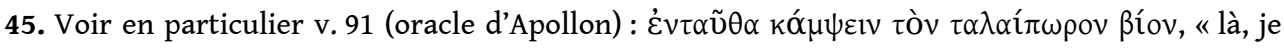

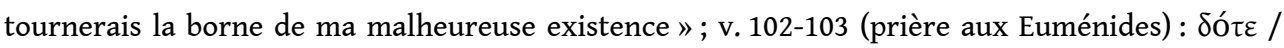

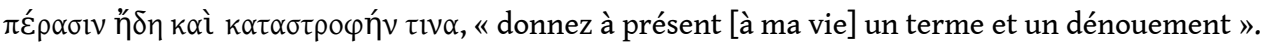

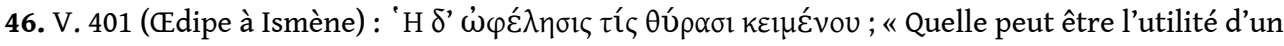
mort étendu à leurs portes?».

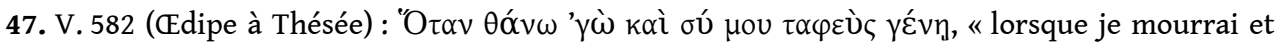
que tu me mettras dans la tombe ».

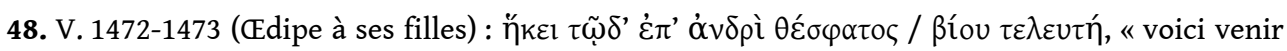
sur moi la fin de ma vie annoncée par les dieux ».

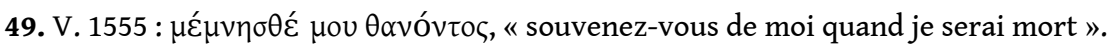

50. Voir en particulier v. 1656-1665.

51. Le texte des manuscrits $\lambda \varepsilon \lambda$ ภı d'éditeurs (entre autres Jebb, Dain, Dawe, Kamerbeek, Avezzù) ne pas permettre d'aboutir à un

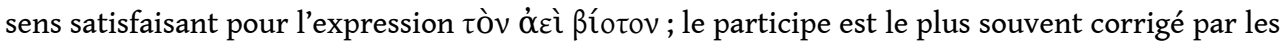

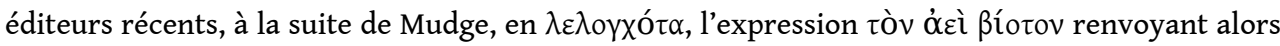
à la vie post mortem d'€Edipe ; pour cette interprétation du texte, voir aussi FERRARI 2003. Le texte transmis par les manuscrits, qui avait été défendu par Hermann, est en revanche maintenu par Lloyd-Jones et Wilson (cf. LLOYD-Jones, WILSON 1990, 261) ; il est défendu par EASTERLING 2006, 139-140.

52. V. 72, ๔

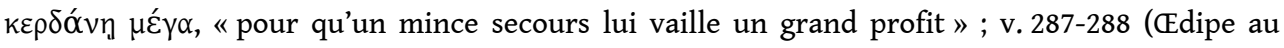

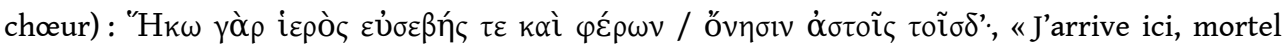
consacré et pieux, apportant un bénéfice à tous ces citoyens ».

53. Tous les passages de la pièce dans lesquels il est question de la présence salutaire du corps d'ÆEdipe sont passés en revue, avec le vocabulaire afférent, par FESTUGIÈRE 1973, 15-20.

54. V. 457-460.

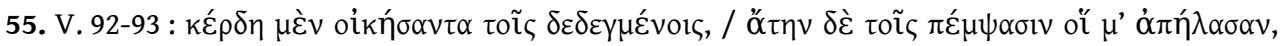
« (là, je deviendrais) un profit, si je m'y installais, pour ceux qui m'auraient accueilli, un fléau pour ceux qui m'ont chassé et mis sur les routes".

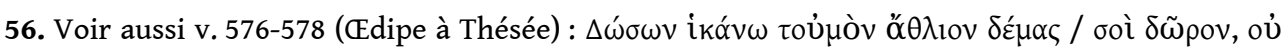

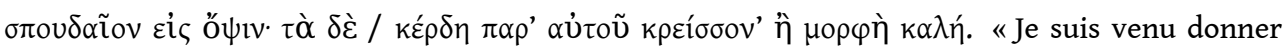
mon pauvre corps, / et le don est pour toi ; à le voir, il ne vaut pas grand-chose ; mais / le profit qu'il représente vaut plus qu'une belle apparence ".

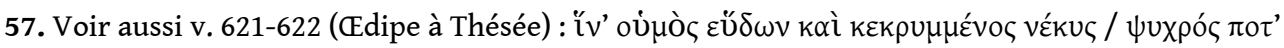

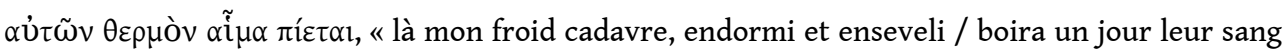
chaud».

58. BLUNDELl 1989 (sur đedipe comme incarnation de ce principe dans đEdipe à Colone, voir en particulier 226-232).

59. C'est bien ainsi en tout cas que l'entend CEdipe, et sa haine va tout autant à Créon qu'à ses propres fils, qu'il rend également responsables de son exil (cf. v. 427-444).

60. Cf. v. 389-392.

61. Comme le souligne l'emploi insistant du verbe $\kappa \rho \alpha \tau \varepsilon ́ \omega$, v. 400, 405, 408.

62. Cf. v. 399-400, 401, 404-405, 784-785.

63. Cf. v. 406-407. 
64. L'élargissement du culte au-delà du cercle familial et son ancrage civique sont en effet des traits bien répertoriés des cultes héroïques.

65. Voir infra.

66. Ce point est bien souligné par SEGAL 1981, 401-403.

67. Sur les enjeux politiques de cette lutte pour la possession de la tombe d'ÆEdipe et sur le rapport entre culte héroïque et protection du territoire, voir KowALzIG 2006, 81-85.

68. cf. v. 784-786.

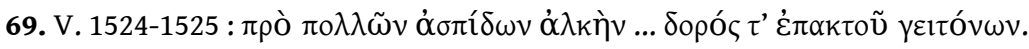

70. Diodore de Sicile 13, 72, 3-73, 2 (le texte mentionne l'Académie, qui est située non loin du dème de Colone) ; voir aussi Xénophon, Helléniques 1, 1, 33-34.

71. Schol. Ael. Arist. 46, 172 (Dindorf III 560).

72. «Il gagne ainsi le seuil à pic dont les assises d'airain s'enracinent dans notre sol. Il s'arrête dans l'un des chemins qui rayonnent de ce point, tout près d'un creux formant cratère, où à jamais se conserve le texte des loyaux serments que se sont prêtés jadis Thésée et Pirithoos. Il s'arrête à distance égale de ce cratère, du roc de Thoricos, du poirier creux et du tombeau de pierre " (trad. P. Mazon). Les éléments de cette topographie sont examinés en détail par EASTERLING 2006, 141-143.

73. Sur la valeur symbolique de cette topographie, voir SEGAL 1981, 369.

74. Cette donnée du secret a été mise en rapport avec un autre référent religieux, celui des Mystères d'Éleusis, qui est également présent à l'arrière-plan d'Ædipe à Colone, comme l'ont montré en particulier CALAME 1998, 349-351; M ARKANTONATOS 2002, 197-220; F ERRARI 2003 ; MARKANTONATOS 2007, 135-140 (sur l'arrière-plan éleusinien des tragédies de Sophocle, voir aussi SEAFORD 1994b, qui porte sur Électre et Ajax). Sans reprendre, après ces critiques, l'énumération des différents passages de la tragédie qui fondent une telle lecture, on peut à tout le moins rappeler qu'il y a une allusion directe à Éleusis et aux Mystères, dans le $2^{\mathrm{e}}$ stasimon, lorsque le chœur imagine les localisations possibles de la bataille opposant les Athéniens et les Thébains (cf. v. 1047-1053). Éleusis est ainsi un lieu qui, comme un certain nombre d'autres dans cette tragédie, fonctionne à la fois comme un point d'ancrage des événements du drame dans une géographie réelle et connue du public, et comme un arrière-plan symbolique qui prend tout son sens dans son rapport avec le destin du héros. Certains éléments relatifs aux circonstances de la mort d'œdipe - en particulier le changement de vêtements auquel procède le héros lors des rites purificatoires (v. 1597-1603), l'éclat de lumière violent qui aveugle Thésée (v. 1650-1652) au moment de sa mort, enfin, le secret qui entoure le lieu de sa disparition (v. 1520-1532) - peuvent en effet se lire comme autant de points de contact avec les rites éleusiniens. Ce n'est pas à dire, à mon sens, que le changement de statut d'CEdipe soit assimilé par Sophocle à une initiation. Mais les mystères d'Éleusis fournissent un modèle analogique qui aide à penser le $\theta \alpha \tilde{u} \mu \alpha$ (cf. v. 1586 :

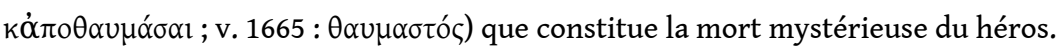

75. CEdipe à Colone, 1518-1534.

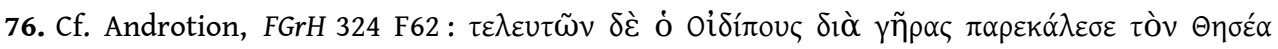

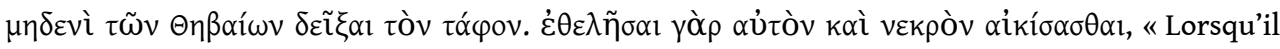
allait mourir sous l'effet de la vieillesse, Ædipe demanda à Thésée de ne montrer son tombeau à aucun des Thébains, parce que, disait-il, il (sc. Créon) voulait le maltraiter, même à l'état de cadavre ».

77. Fr. 65 Austin (= fr. 370 Kannicht), en particulier v. 71-74 et v. 83-89 (AUSTIN 1967) ; voir LACORE 1995-1996.

78. Plutarque, Sur le démon de Socrate, $578 \mathrm{~b}$ : «À son retour, il (sc. le Spartiate Lysanoridas) a

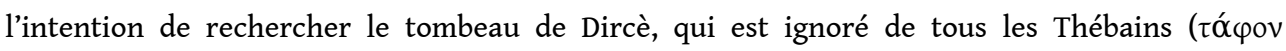

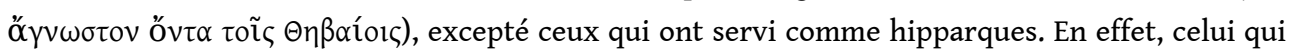
sort de charge emmène son successeur pour lui montrer le tombeau; ils sont tous deux seuls 


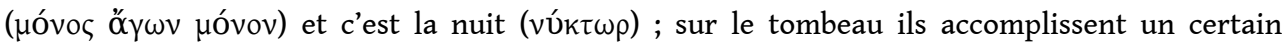

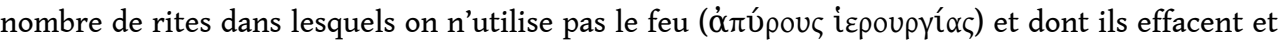

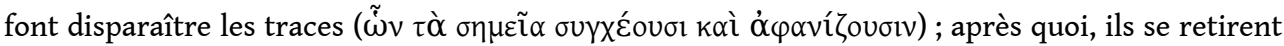

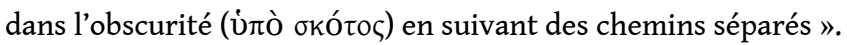

79. Pausanias 2, 2, 2 : «Les tombes de Sisyphe et de Nélée - car on dit que Nélée vint à Corinthe, mourut de maladie et fut enterré près de l'Isthme -, je ne pense pas que quelqu'un les chercherait après avoir lu Eumèlos. Car il dit que même à Nestor, Sisyphe ne montra pas la tombe

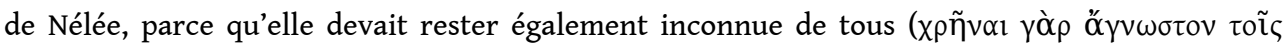

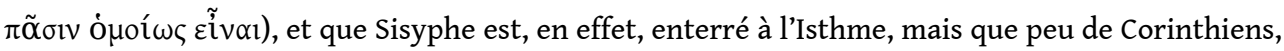
même ceux de son temps, savaient où se trouvait la tombe ".

80. Il est dit simplement que le sanctuaire des filles d'Érechthée, dont l'établissement est décidé par la déesse Athéna, à la fin de la tragédie, doit être frappé d'un interdit religieux (abaton), de manière à ce que les ennemis ne viennent pas y faire des sacrifices en cachette, dans l'espoir d'obtenir la victoire à la guerre.

81. VIAN 1963, 106 suggère que Sophocle s'est inspiré de la légende thébaine de Dircè. KeARns 1989,52, n. 35 est sceptique (« there are some genuine parallels between Attic and Boeotian cultforms, and it is also an open question how many Athenians would have been aware of the details of the Theban cult of Dirke »).

82. HENRICHS 1983 propose d'interpréter la mention que fait CEdipe de son abstention de vin (v. 100 : vท́ $\varphi \omega v)$ comme une allusion à son culte futur, dont les modalités seraient calquées sur celui des Euménides; si l'on admet cette interprétation, force est d'admettre que l'allusion est des plus ténues; il n'y a pas de référence assurée à un culte d'đEdipe dans le reste de la pièce.

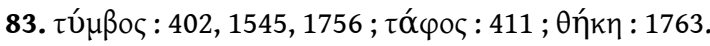

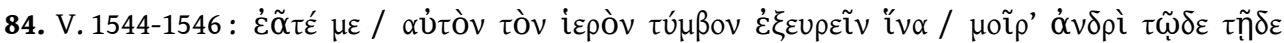

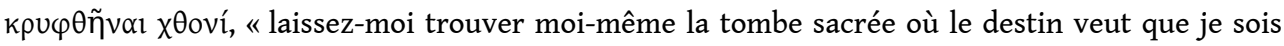
enterré dans ce pays ».

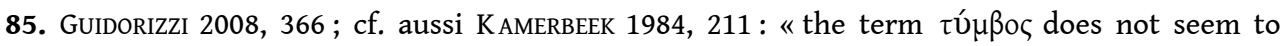
signify more than the place where he is to disappear, nor, of course, does Antigone's use of the term 1756 mean more than that she simply cannot speak of it in other terms $»$.

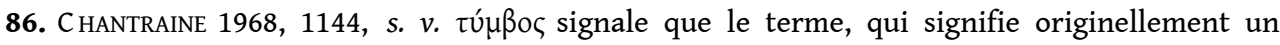
"tumulus funéraire», "est devenu un terme général désignant toutes sortes de chambres funéraires ".

87. Cf. v. 1403-1406: Teucros donne des ordres à ses compagnons pour qu'ils creusent la fosse destinée à recevoir la dépouille d'Ajax et pourvoient aux préparatifs du rite funèbre.

88. Le mot $\tau \alpha \varphi \varepsilon u ́ \varsigma$ est un hapax de Sophocle, que l'on trouve aussi dans Électre (v. 1488), où il est employé ironiquement pour désigner les oiseaux et les chiens, dans leur office de charognards.

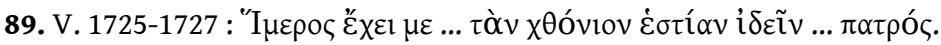

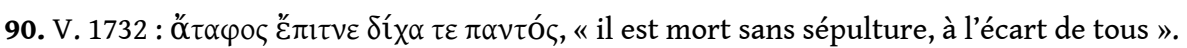

91. KAMERBEEK 1984, 231 : « 'without burial' rather than 'without tomb'».

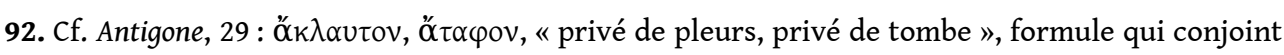
l'absence de rite funèbre (symbolisée par l'absence des larmes versées par les proches du défunt) et l'absence de tombeau ; c'est à propos de Polynice que l'adjectif ö $\tau \alpha \varphi \circ \zeta$ est le plus souvent employé dans les textes grecs ; pour un exemple différent, voir par ex. Hérodote 9, 27.

93. L'originalité de cette version de la mort d'ÆEdipe ressort encore davantage si on la compare à la tradition suivie par Sophocle dans Antigone (cf. v. 897-902), selon laquelle Antigone a rendu les honneurs funèbres à son père, ainsi qu'à sa mère et à son frère Étéocle, conformément au devoir qui incombe aux femmes de la famille lors des funérailles de leurs proches.

94. Cf. v. 1647-1649.

95. V. 1661-1662. 


\section{RÉSUMÉS}

L'đEdipe à Colone de Sophocle est, de toutes les tragédies conservées, celle qui offre le plus de matière à une réflexion sur le phénomène de l'héroïsation en contexte tragique. De fait, toute la tragédie préfigure et dramatise l'accession finale d'ÆEdipe au statut de héros. L'accord ne se fait cependant pas dans la critique sur la question de savoir si, et jusqu'à quel point la mort mystérieuse d'œEdipe dans le bois sacré des Euménides doit être mise en rapport avec les données de la religion grecque. C'est à ce débat que l'exposé suivant voudrait contribuer, en confrontant les données de la pièce, d'une part avec les traditions antérieures relatives à la mort et aux cultes d'œdipe, d'autre part, avec les composantes fondamentales du culte héroïque. Nous chercherons à montrer que, si les realia religieux informent certains éléments de la représentation du destin post mortem d'œdipe, les références ponctuelles aux réalités du culte héroïque soulignent en définitive à quel point la mort d'ÆEdipe est un événement irréductible à tout modèle. C'est finalement beaucoup moins l'instauration d'un culte que l'accomplissement d'un destin singulier qui est ainsi donné à voir.

Can Oedipus' journey from life to death, as it is dramatized in Sophocles' Oedipus at Colonus, be interpreted in the light of Greek hero cult? Should the intriguing narrative of the messenger, telling of the mysterious disappearance of the hero in the sacred grove of the Eumenides, be read as an aetiology of Oedipus' cults in Attic? No consensus exists among scholars about these questions and probably no definitive answer can be given to them. The aim of this paper is to contribute to the debate by confronting the Sophoclean representation of Oedipus' death and posthumous existence, first, with previous traditions related to EEdipus' death and cults, and secondly, with key features of hero cult. We will try to show that, while building on the religious experience of his public to arouse in it an intellectual and emotional response to the mysterious death of the protagonist, Sophocles wants us to understand Oedipus' heroization above all as a dramatic event and the fulfillment of a unique destiny.

\section{INDEX}

Mots-clés : đdipe, Sophocle, héros, héroïsation, culte héroïque

Keywords : Sophocles, Oedipus, hero, heroization, hero cult

\section{AUTEUR}

\section{CHRISTINE MAUDUIT}

ENS-PSL, AOrOc

École normale supérieure

45 rue d'Ulm

75005 Paris

christine.mauduit(at)ens.fr 